\title{
VIVIR A DESTIEMPO. HACIA UNA TEMPORALIDAD IRREVERENTE DE VIVIR CON DOLOR CRONIFICADO
}

\section{Living Untimely. Towards an Unapologetic Temporality of Living with Chronic Pain}

\author{
Dresda Emma Méndez de la Brena* \\ Universidad de Granada
}

\section{Palabras clave \\ Vivir a destiempo Temporalidades tullidas \\ Fibromialgia Dolor cronificado Normativo}

\section{Keywords}

Living untimely Crip temporalities Fibromyalgia Chronic pain Normative

\begin{abstract}
RESUMEN: El presente artículo propone el concepto de "vivir a destiempo" a partir de las reflexiones de Sora, mujer de origen marroquí, diagnosticada con Fibromialgia. A partir de un análisis crítico de la temporalidad desde los estudios contemporáneos queer y crip, se explora el concepto de "vivir a destiempo» como categoría de análisis útil para evidenciar el régimen temporal normativo que excluye de condiciones sociales y materiales a cuerpos con dolor cronificado que no se ajustan a las imposiciones temporales de las sociedades modernas. En ese sentido, "vivir a destiempo" se sitúa como una temporalidad disidente que transgrede los marcadores del tiempo normativo a través de expresiones temporales inoportunas, inadecuadas e inesperadas. La metodología cualitativa narrativa, la utilización de la fotografía y otras expresiones artísticas permiten articular el concepto de "vivir a destiempo" como una estrategia temporal y espacial con potencial crítico y, a la vez emancipador, que abre posibilidades políticas de vivir irreverentemente el tiempo.
\end{abstract}

ABSTRACT: This article proposes the concept of "living untimely» based on the reflections of Sora, a woman of Moroccan origin, diagnosed with Fibromyalgia. Through a critical analysis of temporality from contemporary queer and crip studies, the concept of "living untimely» is explored as a useful analytical category to demonstrate the normative temporal regime that excludes bodies with chronic pain from material and social conditions; bodies that do not adjust to the temporal impositions of modern "healthy" societies. In this sense, "living untimely» is found as a dissident temporality that crosses the markers of normative time through inopportune, inappropriate and unexpected temporal expressions. Two research techniques - qualitative narrative approach, and the use of photography and other artistic expressions- allow us to propose the concept of "living untimely" that can be articulated as a temporal and spatial strategy with critical, as well as emancipatory, potential, since it opens possibilities of living time irreverently.

\footnotetext{
* Correspondencia a / Correspondence to: Dresda Emma Méndez de la Brena. Universidad de Granada, Instituto Universitario de Estudios de las Mujeres y de Género. Rector López Argüeta, s/n (18071-Granada) - dresda@correo.ugr.es - http://orcid.org/0000-0002-8642-2647.

Cómo citar / How to cite: Méndez de la Brena, Dresda Emma (2020). Vivir a destiempo. Hacia una temporalidad irreverente de vivir con dolor cronificado. Papeles del CEIC, vol. 2020/2, papel 235, 1-18. (http://dx.doi.org/10.1387/pceic.20969).
}

Fecha de recepción: junio, 2019 / Fecha aceptación: marzo, 2020

ISSN 1695-6494 / CC 2020 UPV/EHU 
Conocí a Sora en Septiembre de 2017 en la Unidad de Salud Mental en el Hospital de Traumatología en la Ciudad de Granada como parte de mi trabajo de investigación doctoral'. Sora es una mujer marroquí de 54 años que ha vivido con dolor cronificado desde los 15 años de edad, siendo diagnosticada a los 34 años con Fibromialgia. La Fibromialgia es una forma de malestar corporal en donde el dolor experimentado no se corresponde con signos de una enfermedad orgánica, por lo que es un enigma para la investigación biomédica y biopsiquiátrica (Mora, Pujal i Llombart y Albertín, 2017). Para poder entender las especificidades físicas y emocionales de su dolor, Sora junto con otras 15 mujeres con Fibromialgia, asistía mensualmente a una sesión de grupo terapéutica dedicada a abordar la relación entre el cuerpo, el dolor y las emociones.

Me uní a las reuniones ya tarde en el proceso de reflexión colectiva. Muchas de las participantes llevaban asistiendo hacía más de un año, como es el caso de Sora. Mi posición como investigadora dentro del grupo fue observación participativa. Me sentaba en círculo, escuchando y tomando notas de las experiencias y los significados del dolor que las participantes construían colaborativamente. Por las propias características del grupo, la asistencia era intermitente. Las causas más comunes de ausentismo eran cuestiones de salud, citas médicas previamente agendadas o por un tema de cuidados a dependientes. La única vez que Sora no pudo asistir a una sesión fue justamente por esta última. Sora tuvo que ir a Ceuta para cuidar de su madre enferma.

Durante las sesiones, Sora siempre se mostró reflexiva y participativa mientras narraba fragmentos de la historia de su vida. Historia que, como veremos, es un entramado de intersecciones de género, raza, clase y religión que producen y estructuran la experiencia de su dolor. Un mes antes de su ausencia, le había entregado una cámara fotográfica desechable para que capturara los momentos, espacios y objetos que ella relacionaba, tanto de manera positiva como negativa, con su dolor. En total, de las 5 cámaras que se repartieron en este grupo, sólo se recuperaron 3; entre ellas, la de Sora.

En mayo, retomé el contacto con Sora y en junio de 2018 tuvimos nuestra primera entrevista. Quedamos en vernos en la terraza de su cafetería favorita. Con una taza de café y un par de cigarros (fumar es un secreto para su familia por cuestiones religiosas), conversamos a lo largo de una hora y media sobre su trayectoria vital y la experiencia con dolor cronificado. Yo dejo mi grabadora a un costado de su taza de café y me dejo absorber en la conversación. En la grabación se pueden escuchan los pequeños golpes de la cuchara que Sora da en el borde de la taza mientras revisa las fotografías que se desprenden del fotograma. Sora coge la fotografía numerada 25A, la cual claramente le cautiva la mirada desde un inicio. Yo le pregunto qué significados tiene esta fotografía para ella; y así, sin más, con una voz calmada y elegante, Sora comienza su historia.

1 Este artículo forma parte del proyecto «Género, emociones y subjetividad en las relaciones entre pacientes y profesionales sanitarios. Un acercamiento interdisciplinar» que coordina la Dra. Rosa Medina Doménech (Universidad de Granada) y financiado por la Agencia Estatal de Investigación (AEI) y el Fondo Europeo de Desarrollo Regional (FEDER). Agradezco también a la Dra. Ana Távora por las facilidades otorgadas para la asistencia a sus sesiones terapéuticas grupales. 


\section{EL TIEMPO DE SORA}

Destiempo: Loc. Ad. Este vocabulario hace referencia como fuera de tiempo, de un modo o manera inoportuna, imprevista, improcedente, inesperada e inopinada, sin conveniencia, coyuntura, casualidad, oportunidad, congruencia, conformidad, ocasión y exactitud.

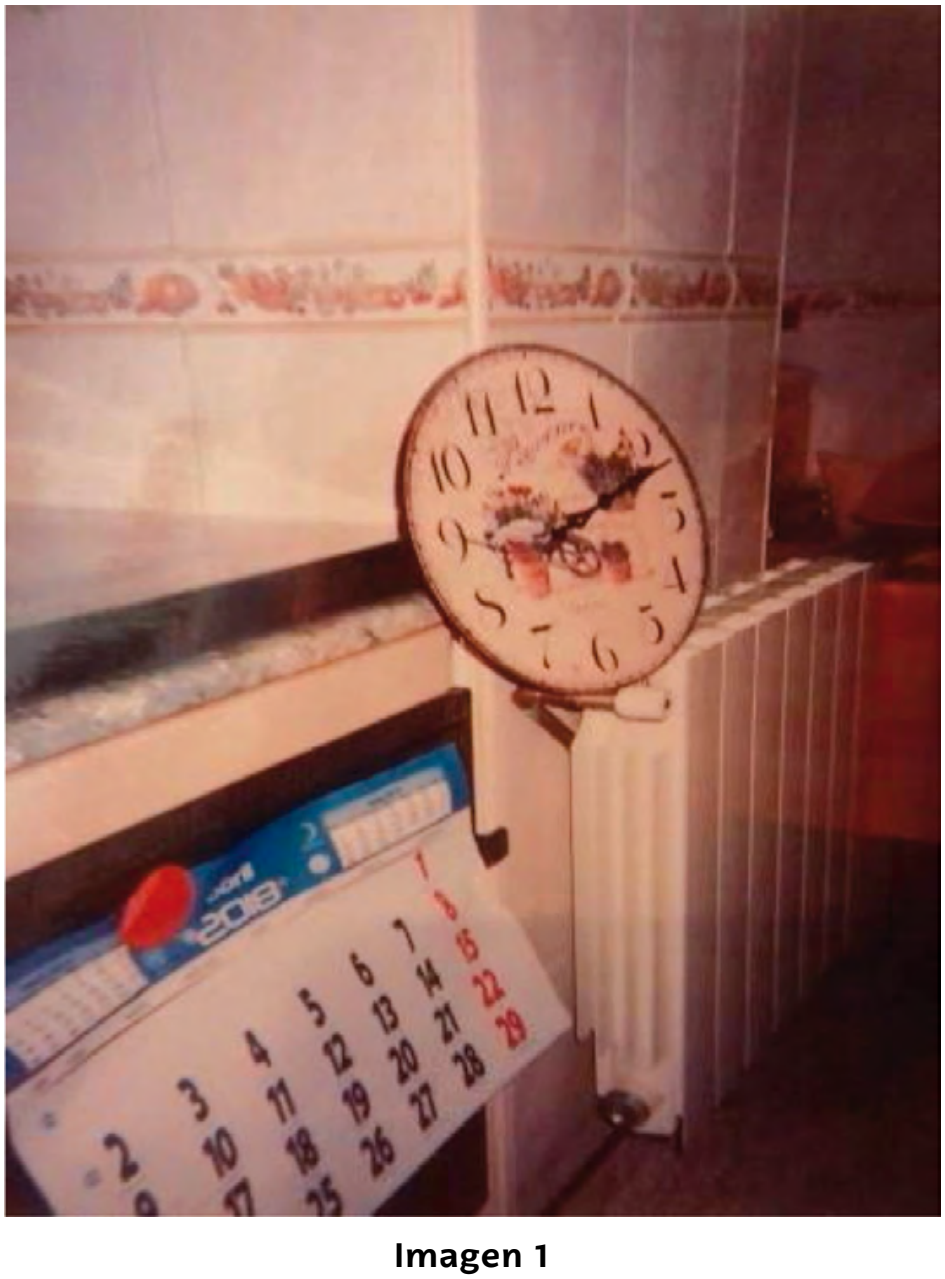

Sora, foto 25A. Reloj. 2018, $10 \mathrm{~cm} \times 15 \mathrm{~cm}$, papel

Este [refiriéndose a la fotografía] es el tiempo. El tiempo es como que no me deja ser yo. Es como que en cada momento de tu vida tienes que estar en un sitio o en otro. Pero para mí, el tiempo es una prisión. Yo no vivo en este tiempo y estoy condicionada a un tiempo que me han inventado. Los doctores me han estructurado la vida: cuando comer, cuando tomar medicamento, cuando ir a consulta. Pero es que en realidad, yo vivo a destiempo y esto implica otra forma de determinarme. Yo vivo a destiempo, y no es que quiera hacerlo, pero vivo en otra temporalidad. Yo no puedo decirte mañana quedamos a las 4 de la tarde porque puede ser que mi cuerpo no me llegue. Es como para esta entrevista, te dije entre las 2 y 3 de la tarde para que me esperaras porque yo no sabía si me alcanzaría el cuerpo para llegar. Luego me pregunto ¿por qué tengo que vivir en la tensión de un tiempo que no es el mío? Yo no sé en qué día estoy. Yo tengo un almanaque en la nevera, un calendario en el cuarto, un reloj en mano. Pero ya no, con el tiempo yo no puedo. Yo no soy una anárquica, pero yo no puedo con este tiempo y he dejado de vivir en él. 
El relato nos adentra en la experiencia cotidiana de Sora con el tiempo. Un tiempo inventado por los esquemas biomédicos y reforzado por las imposiciones y demandas temporales que la condicionan y mantienen su cuerpo en aprisionamiento. Sí, un cuerpo como aquel que mencionaba el filósofo francés Michel Foucault (2012). Aquel que es vigilado, castigado y controlado; aquel que solo se convierte en fuerza útil en tanto que es productivo y sometido, ya no necesariamente a través de fuerzas visiblemente violentas sino ahora por técnicas susceptibles y discretas como el simple tic-tac de un reloj.

La fotografía de Sora acompaña la potencialidad de su testimonio. El encuadre del reloj sobrepuesto junto al almanaque es un representación visual del régimen temporal artificial que actúa sobre su cuerpo, violentándolo y manteniéndolo tirante dentro de las imposiciones temporales que la fuerzan a ajustarse a ritmos biológicos y sociales normales. Como consecuencia, Sora ha tenido que ingeniar otra temporalidad para negociar diariamente sus capacidades físicas. Esto la sitúa en complejos procesos discursivo-materiales de temporalidad que necesitan ser analizados más allá de las concepciones normativas del tiempo.

En nuestra conversación, Sora introduce la noción de "vivir a destiempo" como una temporalidad que le otorga posibilidades de auto-determinación; es decir, le permite tomar una postura diferente hacia el mundo, precisamente porque da cuenta del lugar desde donde ella se enuncia, su discapacidad (Pujal i Llombart y Mora, 2013), y, con ello, recuperar control sobre su tiempo. No obstante, su reflexión va más allá, ya que nombra y visibiliza las violencias temporales ejercidas sobre cuerpos con dolor cronificado, así como las formas de resistencia que permiten reclamar una política temporal irreverente.

Pensar con y a partir del concepto de Sora es una oportunidad para abordar el estudio del dolor cronificado desde un análisis crítico de las epistemologías tullidas y su relación con la identidad, la abyección física y la discapacidad. Además, permite situar el concepto de «vivir a destiempo" como categoría de análisis útil dentro de los debates académicos actuales sobre temporalidades queer y crip y, como una noción valiosa para articular estrategias temporales de cuerpos disidentes que se oponen al tiempo normativo. Importante mencionar que, tanto el dolor cronificado como el destiempo de Sora, se analizan con una mirada crítica de los estudios feministas y de género. Lo anterior atiende a los sistemas de opresión que les intersectan, generando con ello, locaciones epistemológicas específicas que sitúan la experiencia e intuición de Sora como método de hallazgo epistemológico.

\section{3. "VIVIR FUERA DE TIEMPO» Y TEMPORALIDADES QUEER}

El relato de Sora nos proporciona una clave desde donde comenzar el análisis de la temporalidad: la artificialidad del tiempo. No es casualidad que Sora haya realizado una composición visual situando juntos dos marcadores temporales artificiales: el almanaque y el reloj. Como piezas complementarias, ambas forman parte del engranaje temporal que, como Sora refiere, "le inventan el tiempo" y la condicionan a un ritmo social determinado y a una autorregulación individual (Freeman, 2010). La artificialidad temporal que Sora pone en evidencia ha sido explorada por los estudios queer contemporáneos. Autores como Judith (Jack) Halberstam (2005), Lee Edelman (2004) y José Esteban Muñoz (2009) argumentan 
que el tiempo es fundamental en la producción de la normalidad, denunciando persistentemente las diversas maneras en que las identidades son atravesadas por constructos temporales que orientan la vida a un orden social y sexual que perpetua la "heternormatividad obligatoria»².

Halberstam (2005) por ejemplo, argumenta que los imperativos de la heteronormatividad obligatoria -es decir, la idea de normalización de la sexualidad y su reglamentación en la sociedad - están sostenidos bajo la lógica de temporalidades lineales: nacimiento, matrimonio, familia, productividad, muerte y herencia. Estas lógicas fuerzan relaciones heterosexuales que se institucionalizan y que equiparan por igual a todos los miembros de la sociedad. En este sentido, y como oposición y renuncia a los regímenes heterosexuales, los trabajos de Halberstam (2005), Edelman (2004) y Muñoz (2009) exploran, a partir de experiencias de subculturas queer y otras formas de organización identitaria y/o sexual en los Estados Unidos durante los años noventa, cuestionamientos del tiempo heteronormativo que residen "fuera" de las aprensiones lineares del tiempo. Es decir, formas temporales queer que resisten desde otros lugares a la crononormatividad o mecanismos artificiales de manipulación y regulación corporal (ej. horarios, calendarios o zonas horarias) (Freeman, 2010) y, a la heterofuturabilidad (Edelman, 2004; Sáez, 2017), esto es, a la idea de futuro proyectada por la familia heterosexual.

Ejemplo de esto es el trabajo de Lee Edelman (2004). El autor propone cómo el futuro normativo, en el contexto estadounidense, se piensa a través de términos reproductivos y, cómo esta idealización moldeada a partir de la figura retórica de The Child (El Niño), debería ser rechazada totalmente por las identidades y la teoría queer. La figura de "El Niño» proscribe un futuro en términos de reproducción, generación y herencia con el fin de mantener el sistema de privilegios (blancos, clase media, masculinos, capacitistas) que, bajo la figura simbólica de "El Niño», transmiten su legado. Por lo tanto, la alternativa queer debería ser el rechazo total a la progenie $y$, por tanto, al futuro para así poder cuestionar el presente.

Con el rechazo a la heterofuturabilidad y las operaciones de resistencia de las subculturas queer "fuera" de los marcadores temporales paradigmáticos de experiencia (nacimiento, matrimonio, reproducción y muerte), el «tiempo queer» surge como una propuesta para la desestabilización de regímenes temporales hegemónicos. El «tiempo queer» inauguró una temporalidad de nuevas narrativas de vida disidente y de relaciones alternativas al tiempo y espacios normativos. De esta manera, para las subculturas queer, "vivir fuera de tiempo" se convirtió en una nueva lógica temporal que implicaba la existencia de la temporalidad y la subjetividad "fuera» de las expectativas del tiempo normativo para resistir el aqui y ahora (Muñoz, 2009) y, con ello, desafiar el proyecto temporal homogeneizador. Sin embargo, la teórica Alison Kafer (2013) menciona que, aunque los discursos del tiempo queer se construyen a través de la referencia a la enfermedad (Halberstam, 2005) y la muerte (Muñoz, 2009; Edelman, 2004) como marcadores temporales, en el fondo no logran complejizar el tema de la discapacidad y su temporalidad.

2 La conceptualización de Adrien Rich en 1980 sobre la heterosexualidad obligatoria fue precursora importante del concepto de heteronormatividad. Rich (1996) cuestionó la práctica de aceptar la heterosexualidad como algo normal, resultado esperado del desarrollo, de nunca cuestionar cómo se desarrolla una "preferencia» para una pareja del sexo opuesto, mientras se patologiza y cuestiona arbitrariamente el desarrollo de aquellos que se sienten atraído/as por parejas del mismo sexo. 
No es que las temporalidades queer y crip trabajen de manera separada -claramente ambas incorporan en su análisis identidades y cuerpos disidentes y prácticas no normativas-, pero al leerlas en relación con sus propias áreas de estudio, existen también desconexiones. Por ejemplo, Kafer (2013) argumenta, a partir de su crítica a Edelman, que los discursos de reproducción, generación y herencia están llenos de una ansiedad por la discapacidad que no expresa el autor en su obra. Un futuro reproductivo es aquel que proporciona las condiciones en donde "El Niño" (blanco, varón, de clase media) sea saludable y, en un futuro como ese, "los niños discapacitados no son parte de este imaginario privilegiado excepto como el otro abyecto" (ibídem: 33). Por ello, comenta Kafer (2013), el abandono del futuro que propone Edelman, no es una opción viable para los estudios de la discapacidad porque sería negar que algunos futuros se encuentran más protegidos que otros. Se necesita, entonces, buscar temporalidades alternativas que no expulsen a las personas con discapacidad fuera del tiempo, sino que den espacio para la coincidencia y la diferencia.

Volver al relato de Sora puede ayudar a ilustrar la necesidad de buscar temporalidades alternativas ante las complejidades específicas de cuerpos no normativos, especialmente de aquellos que necesitan asistencia. En el fragmento que se presenta a continuación, Sora comparte las dinámicas que se entretejen en relación con las necesidades de su cuerpo dolorido. Dinámicas que tienen que ver con las relaciones familiares y afectivas, y las configuraciones del tiempo y espacio personal, cuando una de sus integrantes tiene una discapacidad que requiere de cuidados permanentes:

Cómo me explicas cuando te casas con un cuerpo, con unos sentimientos, tienes hijos y ahora, tienes que vivir un matrimonio que ya no solamente no existe pero que por las condiciones de tu cuerpo roto no puedes salir de él.

El matrimonio de más de veinte años de Sora es solo un recuerdo de lo que fue. Aun así, Sora sabe que, dadas las condiciones de su dolor, su cuerpo le demanda cuidados de los cuales ahora depende, y por los cuales, no puede renunciar a este proyecto de vida importante para su sostenimiento. La imposibilidad de renuncia hace insostenible usalirse de la linealidad del hetero-tiempo" (Muñoz, 2009: 25) y esto está relacionado con lo que Mora, Pujal i Llombart y Albertín denominan "contextos de vulnerabilidad de género del dolor cronificado» (2017). Es decir, el dolor cronificado «articulado simultáneamente con contextos sociales con propiedades específicas» (ibídem: 3 ) que la obligan a seguir "dentro» de las lógicas temporales normativas (matrimonio, familia, y herencia, etc.), porque de lo contrario, sus necesidades de cuidado quedarían desatendidas.

Aunado a la situación de cuidados esta la dependencia económica. Cuando su dolor se hizo más fuerte, Sora quedó relegada al ámbito doméstico mientras su marido se encargaba de la provisión total de la familia. Dado que la provisión refiere a la consecución de logros (dinero, éxito, legibilidad social) orientado a la productividad, las relaciones de cuidado adquieren, por otra parte, "un carácter instrumental» (Mora, Pujal i Llombart y Albertín, 2017). Al ser lo doméstico considerado parte del modelo de género tradicional en términos de la división sexual del trabajo, el esfuerzo que se requiere para realizar las labores del hogar no es reconocido, aunque se espera su cumplimiento cotidiano. Suponer la pérdida del trabajo remunerado, no como decisión autónoma sino como consecuencia de su cuerpo enfermo y, por ello, quedarse en casa sin poder cumplir eficazmente con el mandato de género, resulta en la interiorización de sentimientos de fallo que tienen un impacto en la configuración de la subjetividad. Sora comenta: 


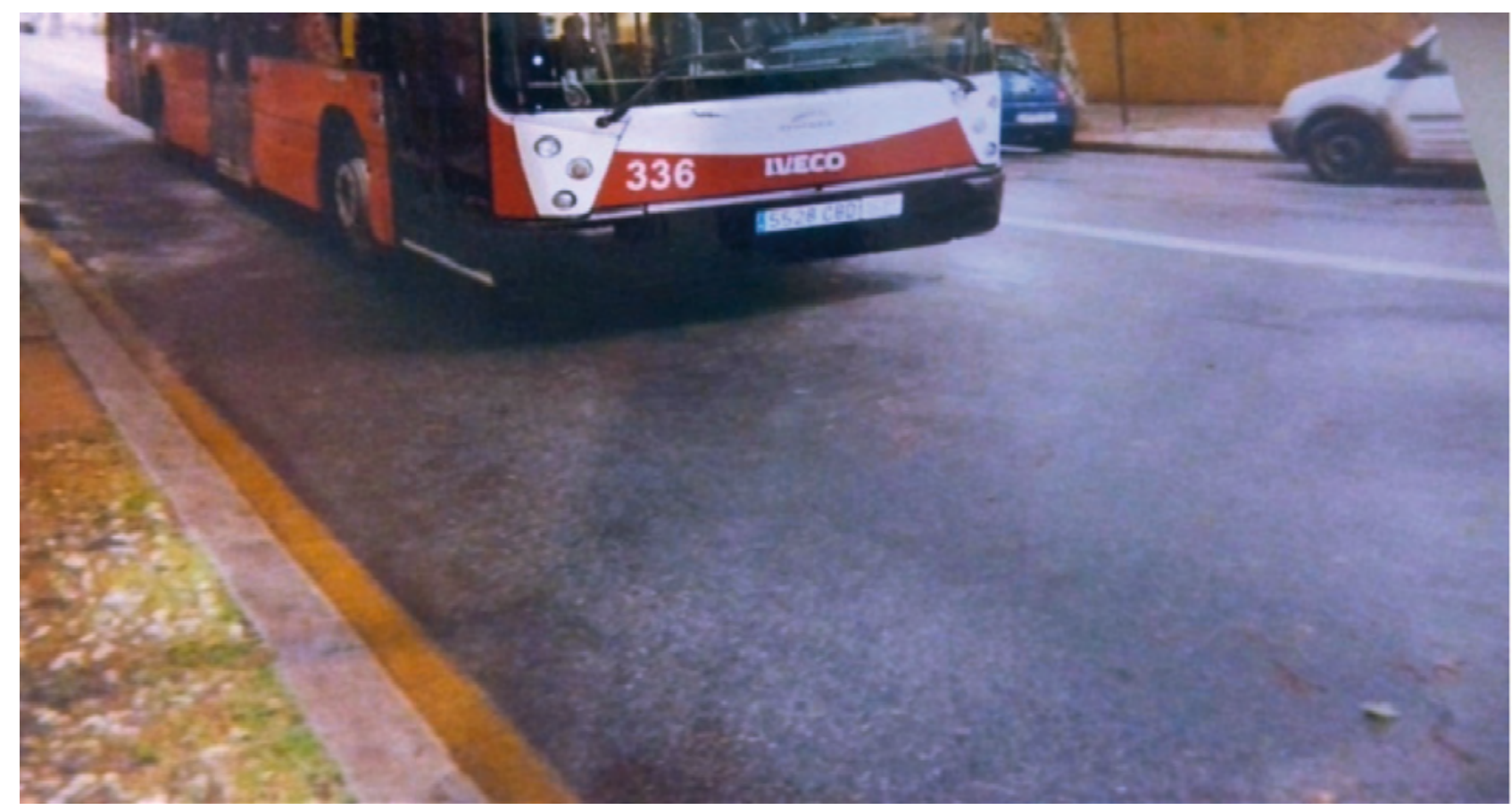

Imagen 2

Sora, foto 17A. Reloj. 2018, $10 \mathrm{~cm} \times 15 \mathrm{~cm}$, papel

Ese número, 336, era el número que tomaba todas las mañanas para ir al trabajo. Cuando mi dolor no era tan agresivo, sabía el tiempo justo que me tomaba llegar a la parada, y mi hija me acompañaba a cogerlo. Hoy, lo único que veo cuando veo ese número es un fallo en mí, un problema en mi cuerpo. Veo pasar el autobús con personas que están a tiempo para llegar a su trabajo. Personas normales, adecuadas para cumplir con los horarios de trabajo. En cambio, yo, cansada, adolorida. Dicen que tenemos la enfermedad del vago. Porque no queremos hacer nada. Yo no soy vaga, pero simplemente no puedo trabajar en ese acomodo del tiempo.

Sora captura la imagen del autobús número 336 que tomaba diariamente cuando iba a trabajar como cuidadora de personas mayores. En su relato, vemos la forma en que su subjetividad se establece entre la dependencia y vulnerabilidad, entre el progreso y el fallo, entre la normalidad y la discapacidad. Sora ya no puede acomodarse a este tiempo, el cual está ligado a la idea de productividad y progreso; $y$, sin embargo, no puede salirse de este, ya que como parte de la múltiple, intensa y continuada demanda por parte de su pareja de, como comenta Sora, "producir algo si es que va a estar sentada en casa», Sora realiza trabajos de repostería en casa diariamente.

Recordemos que la idea de progreso es fundamental para los estudios queer, ya que la propuesta del tiempo queer es salirse de las lógicas temporales normativas: matrimonio, familia y herencia, y con ellas, de la lógica del progreso. Cabría suponer que una temporalidad queer se opone a cualquier noción de progreso y, en términos generales, esta suposición es legítima. Sin embargo, la autora Cua Lim (2009) hace una advertencia relevante sobre el estudio de temporalidades queer provenientes de academias de occidente las cuales, en su opinión, tienden a criticar "el progreso" pero no desde un pensamiento decolonial "sobre el progreso». Esto, menciona la autora, representa una forma de elitismo temporal y excluyente 
que genera un binarismo dentro/fuera de las lógicas temporales para aquello/as que pueden no desplazarse de un lado a otro de este espectro. En cambio, la autora propone el concepto "tiempos inmiscibles» que define como "múltiples tiempos que nunca se disuelven del todo en el código de la conciencia de los tiempos modernos, temporalidades discretas incapaces de alcanzar la homogeneidad o la plena incorporación en un presente cronológico uniforme» (Cua Lim, 2009: 12).

La propuesta de "tiempos inmiscibles» de Cua Lim (2009) se acerca epistemológicamente a la temporalidad "tullida» que analizan las teorías crip, literalmente teoría tullida según Platero (2014). Las epistemologías tullidas - cripistemologies según McRuer (2006) - refieren a una temporalidad de coincidencias, de realizaciones no normativas, y de negociaciones cotidianas moldeadas a partir de la dependencia (Price, 2017; Kafer, 2013; McRuer, 2006). Como vemos en el relato de Sora, cuando la enfermedad llegó y el dolor se hizo más fuerte, tuvo que tejer una temporalidad propia que no la situara dentro/fuera del tiempo, sino una que le brindará formas alternativas para acomodarse a y apropiarse de él. Una temporalidad que le permita entender que su discapacidad, así como cualquier otra forma de existencia, está conformada ontológicamente por estados de dependencia y vulnerabilidad que, en el caso de Sora, parten de contextos de vulnerabilidad de género, lo cual requiere de un nuevo planteamiento de su temporalidad, una que le permita "vivir a destiempo».

\section{TEMPORALIDADES TULLIDAS Y TIEMPOS COEXISTENTES}

La temporalidad de Sora está caracterizada por la necesidad insoslayable de flexibilidad más que de la posibilidad de vivir "fuera" del tiempo. La expresión de "vivir a destiempo" expone la necesidad de negociar la coexistencia de su temporalidad con otras formas temporales que concurren en intersecciones de poder/privilegios/tiempos/espacios normativos. Los estudios de la discapacidad y de teorias tullidas han añadido al análisis de la temporalidad queer otras experiencias de ser y estar que no se adecuan al imaginario universal del tiempo. Temporalidades tullidas que buscan formas de expandirse en el tiempo, a través de cambios de ritmo $y$, en el transcurso de estas transiciones, la posibilidad de tiempos y espacios de improvisación los cuales serán incomodos y sin orden (Kuppers, 2014) y, que probablemente, des-estructurarán nuestra idea fija del tiempo conocido.

En los estudios sobre epistemologías crip existen varios ejemplos de temporalidades incómodas en contextos de confluencia temporal-espacial con escenarios de normatividad. Tenemos por ejemplo, el concepto falling in (caer sobre) de Eliza Chandler (2010) como una forma de exploración sobre cómo el acto de tropezar de un cuerpo normativo conduce a "caer sobre" categorías de identificación y desidentificación. $\mathrm{O}$, Time is out of joint (tiempo fuera de coyuntura) de Freeman (2010), cuando el caer enferma empuja a los cuerpos a un tiempo fuera de lugar. O también el concepto de prognosis time (tiempo de pronóstico) de Sarah Lochlann Jain (2007) como una temporalidad liminal en lugar de una temporalidad de progresión estable y constante a través de las etapas de la vida. Estas temporalidades generan lo que Kafer (2013) denomina "tiempo de anticipación», el cual es «una especie de extraña liminalidad, viviendo siempre en anticipación del momento que aún no ha llegado» (ibídem: 38 ). Es decir, temporalidades que permiten ver al cuerpo normativo que la discapacidad siempre 
está presente. Por ello, las temporalidades tullidas son perturbadoras, porque ponen en evidencia la artificialidad del tiempo y el constructo ideológico sobre el cual se basa la idea del cuerpo, la normatividad.

Provenientes de los estudios queer y crip en Estados Unidos, las temporalidades de Jain (2007), Freeman (2010), y Chandler (2010) son ejemplos extraordinarios de conceptualizaciones temporales que ponen en primer plano experiencias y conocimientos de cuerpos diversos - Jain, académica blanca y queer diagnosticada con cáncer, o Chandler, académica blanca con discapacidad no evidente-, que informan y estructuran el contenido epistemológico de sus temporalidades en un esfuerzo por construir una teoría desde los contextos de vulnerabilidad de cada una de estas experiencias. En esta misma línea, la propuesta de este artículo es aportar el concepto de "vivir a destiempo" como categoría útil para las epistemologías queer y crip, ya que plantea contextos de vulnerabilidad específicos. En el caso de Sora, su destiempo tiene una trayectoria marcada por la violencia de género la cual estructura la experiencia de su dolor y su temporalidad.

\subsection{Entre normas de género y desplazamientos}

Toda temporalidad está constituida por historias. El tiempo es lo que nos conecta, corre a través de cosas y procesos, conjugando las etapas de la vida. Si bien el tiempo es una fuerza activa independiente de la materia (Grosz, 2004: 114), es la materialidad del cuerpo la que recibe su fuerza. Por ello, para entender el destiempo de Sora, hay que considerar su trayectoria vital y sus prácticas de vida cotidiana para asi poder entender la naturaleza co-constitutiva del género. Lo anterior permite visibilizar las formas en las que interviene este elemento en la construcción de la discapacidad de Sora y la experiencia de su tiempo.

El «destiempo» de Sora tiene como punto de partida su desplazamiento a Granada a los 18 años de edad para, utilizando sus propias palabras, "escapar de la violencia y autoritarismo de su padre y de las estructuras sociales y culturales opresivas que enmarcaban su vida en Ceuta». Para Sora, cruzar la frontera entre Ceuta y España fue, y sigue siendo, un momento de inflexión porque es estar en dos mundos, en dos temporalidades distintas que le generan tensión y dolor. Regresar a Ceuta nunca le ha sido fácil porque le supone un exceso de sufrimiento. 0 , al menos, asi lo narra Sora en la fotografía que decidió tomar en la frontera de Ceuta al regresar a casa de su madre para cuidar de ella. Su madre lleva cinco años enferma de cáncer lo que la hace cada vez más dependiente físicamente e inestable emocionalmente, y por ende, en necesidad más constante de los cuidados y atención de Sora.

Las fronteras, parafraseando a Anzaldúa (1987), están configuradas para definir los lugares que son seguros e inseguros. Normalmente, cruzamos las fronteras para desplazar nuestros cuerpos y emociones hacia nuevas geografías. En el caso de Sora, su vida en Ceuta era una pesada mochila llena de "odio, vergüenza, altanería, frustraciones» hasta que construyó un puente a España para sentirse más segura, para dejar atrás la violencia de la que provenía. Sin embargo, los puentes llevan en sus cimientos residuos emocionales porque en "los cuerpos coexisten las identidades de opresiones múltiples» (Moraga, 1988: 1) que se cargan en la espalda y, que en esta fotografía, se le hacen presentes a Sora en el cruce fronterizo. 


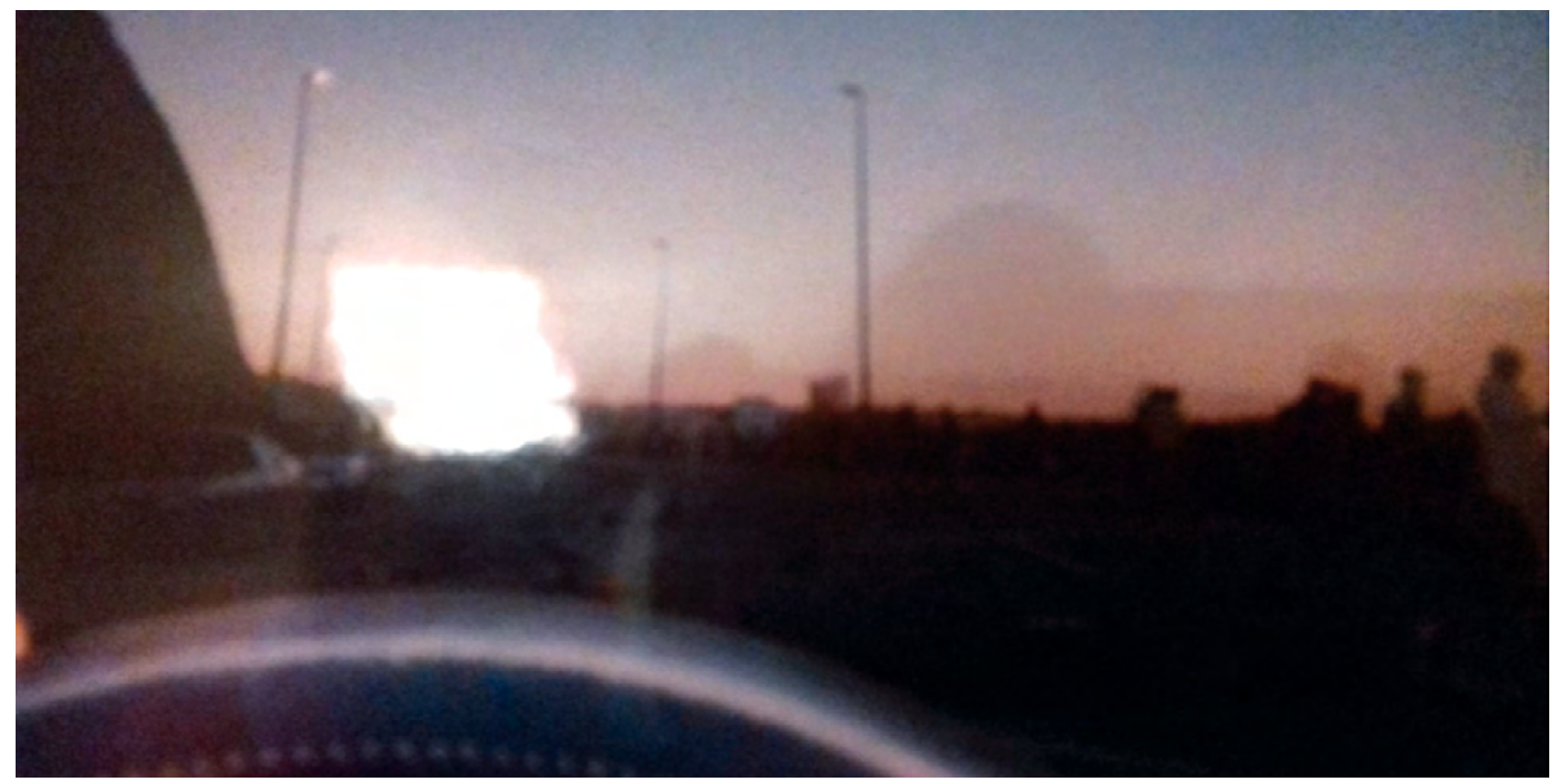

Imagen 3

Sora, foto $12 \mathrm{~A}$. Frontera. $2018,10 \mathrm{~cm} \times 15 \mathrm{~cm}$, papel

Esta foto es de la frontera de Ceuta. Me pillaron y me iban a romper la cámara, por eso no se ve lo que quería que se viera. Todo esto es gente, corriendo de un lado a otro. A veces yo creo que esta frontera puede representar mi conflicto de estar entre dos mundos. Esto quizá es mi niñez, la mochila que yo tenía hasta que no construi mi puente. Hasta que me construí a mi misma como un puente. Todo lo que pasa en esta frontera son mis sentimientos: odio, vergüenza, altanería, frustraciones, aqui hay mucho dolor. Aqui ves el inframundo.

A partir de esta fotografía, Sora y yo comenzamos hablar de su vida en Ceuta y de las diversas situaciones que le hicieron tomar la decisión de migrar a la península. El siguiente relato nos muestra las barreras culturales, sociales y religiosas que quiso dejar atrás. Sora narra un deseo de desplazamiento para romper con todos los significantes que hasta ese momento daban forma a su identidad (su hogar, familia, educación, religión y su condición de género).

S: Yo llegué a Granada con 18 años. Yo acabé el Instituto allí en Ceuta y me vine.

D: ¿Cómo decidiste venirte a Granada?

S: Bueno, lo luché, lo luché porque mi padre ni a sol ni a sombra quería que me viniera.

D: ¿Qué te ánimo para irte de ahí?

S: Yo desde pequeña tenía claro que quería salir de casa y de Ceuta. Me asfixiaba, con la situación de Ceuta, no lo aguataba. No podía, no podía. Con la situación de Ceuta yo nunca pude.

\section{S: ¿Qué pasaba allí?}

D: Primero, la religión en sí misma. La religión de mi casa y la opresión de mi madre. Como vivía mi madre, yo no quería vivir como ella. Mi madre fue una mujer maltratada que vivió así hasta que mi padre falleció. Yo no quería eso para mí. Yo sabía 
que tenía que irme con la cabeza alta o salir escapándome. Cuando acabe la selectividad mi padre me dijo que como mujer no podía salir, pero mi abuelo se puso de mi lado y le dijo "o se va con tu permiso o se va sin él». De ahí, tenía claro que yo a Ceuta no volvería.

D: ¿Sientes que tu dolor disminuyó una vez que saliste de Ceuta?

S: Yo soy una persona reincidente. Salí de la casa de mis padres y me fui con las monjas. Así que salí de una religión y me metí a otra. Siempre he tenido la necesidad de buscar algo que le dé sentido a mi vida. Yo no concibo la vida solo material. Para encontrar una paz interna necesito algo espiritual. Yo de lo que huía era de la tradición, no de la religión. Yo no rechazaba la religión. Rechazaba los palos de mi padre y la represión de las mujeres, por la forma de actuar de los hombres. Pero me acerqué al Islam nuevamente, y me di cuenta de lo que era lo que mi me llenaba. El cura que me tocó de guía me dijo antes de tomar los votos que yo no era feliz. «Hay muchas maneras de servir al mundo porque a ti los hombres te gustan». Me puso en contacto con un grupo de musulmanes que habian aceptado la religión, y gracias a eso encontré el Islam otra vez. Aunque esto para mí fue otra ruptura emocional y religiosa, porque las monjas me dieron la espalda. Puedes pensar que estoy loca. Necesité mi tiempo para encontrarme. Yo en Ceuta no era feliz.

En la historia de Sora hay una trayectoria de procesos vitales individuales en diferentes encrucijadas sociales, culturales, religiosas y de género. Salirse de su casa significaba una oportunidad para romper con patrones de violencia que no quería para sí misma. Al llegar a la península, su primer acto fue cuestionar el significado y significante que durante toda su vida habian conformado su subjetividad, la religión. Sora decide dejar el Islam, el cual consideraba, en un primer momento, opresivo y permisivo de la violencia y opresión de género, buscando resguardo en un convento de monjas agustinas para habitar lugares simbólicos y materiales diferentes en donde le fuera posible pensarse más allá de su historia de represión y violencia.

Durante muchos años Sora vivió al resguardo del convento, pero su inquietud por el mundo le hizo entender su vida más allá del celibato. Años más tarde, decide reencontrarse con el Islam $y$, con ello, abrirse a una nueva comunidad musulmana en donde encuentra la espiritualidad $y$, a quien hasta hoy, es su pareja. Sora se casa a los 25 años, pero su matrimonio no es mejor que el que su madre. Después de varios años de vivir con muchos conflictos de pareja y, peor aún, cuando su hijo mayor comenzó con problemas de drogadicción, a Sora se le rompió definitivamente el cuerpo. Los problemas de su familia, los cuales ella sostenía a discreción, se convirtieron en un silencio ensordecedor para su cuerpo. A razón de esto, le preguntó a Sora si cree que todo el trayecto de su vida tiene un impacto sobre su dolor. A lo que ella contesta:

Para mí realmente [la fibromialgia es] el machaque de la mujer. Yo creo que realmente no es una enfermedad de hoy. Es un fantasma que ha acompañado a las sociedades que ha habido. Porque yo hago recuerdo de pequeña y ver a mi abuela y a otras mujeres machadas, agotadas, que les dolía todo, y ni siquiera iban al médico. Y tiraban y tiraban. Yo creo que es por el gran peso que se les ha puesto a las mujeres encima y que nosotras hemos asumido este papel, tirar de la familia, de la economía, del que dirán, de «eres el honor». La herencia que hemos adquirido las mujeres de hoy día. No digo que sea la verdad, pero viendo la historia de la mujer y el peso que se ha puesto a la mujer encima y el peso que hemos asumido y hemos querido perpetuar, entiendo que nos pase a nosotras. Los hombres son más simples. Su papel en la historia era ir a cazar, guerrear, hacer esta función y ya estaban cumpliendo, pero la función de la mujer nunca ha estado delimitada, el peso nunca acaba. Tienen a los hijos, pero eso significa seguir tirando, el padre trae el sustento 
y para, pero una tiene que ser la perfecta madre, la perfecta hija, la perfecta esposa. Lo peor es que nosotras nos lo creemos y las qué hemos tenido la actitud de preservar esta herencia, estamos marcadas. Las que tienen claro que hacer y qué no hacer, dicen que no, y no están afectadas por esta enfermedad.

Para Sora, la Fibromialgia es parte de una genealogía de opresiones y explotaciones de género. Su dolor está relacionado con los marcadores temporales heteronormativos de los que habla Halberstam (2005). Sora entiende el dolor cronificado como parte de esta lógica temporal lineal de opresión de género en la familia, en el matrimonio y en lo productivo. Por ello, no concibe a la Fibromialgia como una enfermedad moderna sino como parte de una temporalidad heteronormativa que machaca el cuerpo de las mujeres, conservada en forma de herencia y transmisible genealógicamente.

La visión de Sora sobre su dolor se inscribe dentro de investigaciones elaboradas por áreas de conocimiento provenientes de las Humanidades, Estudios Feministas y de Género y Estudios Culturales para identificar elementos explicativos de este malestar, el cual "expresa la desigualdad de género de nuestra sociedad" (Mora, Pujal i Llombart y Albertín, 2017: 3) y, cuyo abordaje en el sistema biomédico, es claramente insuficiente. La emergencia de nuevas formas de malestares corporales (dolores crónicos, fatiga crónica, sensibilidad química múltiple) forma parte del agotamiento normalizado provocado por la presión del mandato de género y por las dinámicas de desigualdad en la economía del sistema neoliberal contemporáneo (Valverde, 2015). La antropóloga feminista Kaja Finkler (1994) denomina a estos nuevos malestares corporales «lesiones de vida»; es decir, malestares ocasionados por contextos de vulnerabilidad cuando existen "contradicciones no resueltas y las indignaciones morales se inscriben en el cuerpo, lo que resulta sintomáticamente en malestar general, dolor y sufrimiento" (ibídem: 16); malestares que afectan de forma mayoritaria a las mujeres. Por ello, la propuesta de Mora, Pujal i Llombart y Albertín (2017) al hablar sobre «dolor cronificado" en lugar de "dolor cónico" para nombrar las complejidades de las relaciones sociales desiguales y los contextos de vulnerabilidad de género que "producen» el dolor, mientras que hablar de dolor crónico da por sentado un malestar de origen orgánico extraído de un contexto social.

El dolor cronificado de Sora responde entonces a un contexto de vulnerabilidad basado en un orden de género normativo y en desigualdad jerárquica entre hombres y mujeres $y$, por consiguiente, su temporalidad tiene una marca de género. Por ello, el posicionamiento de «vivir a destiempo" no solamente revela las estructuras normativas del tiempo sino la aparición de problemas de salud en un contexto de vulnerabilidad de género que los posibilitan. Sin embargo, Sora no es una espectadora pasiva de su tiempo. Como hemos visto en su historia, Sora es una mujer resiliente, nunca tranquila, siempre buscando formas de interpelar(se), por ello, ha encontrado en su «destiempo" una temporalidad que le permite detenerse y recolocarse ante temporalidades prescriptivas de cuidado, tanto familiar como biomédico. Es decir, aquellas temporalidades sostenidas por una visión del tiempo orientada hacia la procuración de progreso.

\section{5. «VIVIR A DESTIEMPO» Y TEMPORALIDADES DE RESISTENCIA}

Partir de los contextos de vulnerabilidad que atraviesan la trayectoria vital de Sora ha sido fundamental para comprender las relaciones entre el cuerpo, el dolor y la temporalidad. $R e-$ 
sultado del análisis contextual observamos cómo el dolor cronificado de Sora es consecuencia de un continuo disciplinamiento temporal del cuerpo y de su sujeción a las normas de género, las cuales no ha podido rechazar completamente. No obstante, a través de sus fotografías e historia de vida podemos ver que, aunque al margen de una completa intencionalidad, Sora se ha permitido en circunstancias y coyunturas temporales concretas habitar su destiempo y legitimar su experiencia del dolor. Sora ha necesitado buscar temporalidades alternativas que no la expulsen fuera del tiempo lineal ni de las instituciones normativas sobre las cuales se sostiene, ya que su cuerpo con dolor, necesita de modos afectivos y de cuidado que dependen de esos lugares. Por ello, Sora ha buscado una temporalidad que le permita un tiempo en coincidencia a través de pequeños actos de resistencia y contestaciones temporales a través de su experiencia corporal.

De esta manera, Sora introduce la categoría de "vivir a destiempo" como una temporalidad de determinación propia, que genera formas temporales agenciales que, a través de la expresión de formas corporizadas inoportunas, inadecuadas e inesperadas, se oponen a las normas desde su discapacidad. "Vivir a destiempo" da cuenta de formas temporales «inmiscibles» (Cua Lim, 2019) como arrastrarse, pausar, guardar silencio, tomarse el tiempo, derrumbarse, llegar tarde, no llegar. Temporalidades que desbordan las expectativas normativas de la temporalidad y la corporalidad. A continuación presento un ejemplo de estas formas temporales de resistencia resultado de una de las fotografías del ejercicio visual realizado por Sora.

En esa fotografía vemos la forma en que Sora se reapropia del "descanso" como una temporalidad que le permite situarse ante los demás desde su discapacidad. La ejemplificación de esta forma temporal se desarrolla en conversación con el trabajo visual narrativo de la artista estadounidense Jennifer Brea en el documental titulado Unrest (2017). La vinculación de ambos trabajos reside en su capacidad "especulativa» (Haraway, 2013); es decir, en su capacidad de generar imaginarios como una práctica activa para concebir nuevas posibilidades.

En los estudios sobre la discapacidad es indispensable poner en práctica la especulación. En concreto, para los estudios de las temporalidades tullidas no se trata solo de añorar una creación totalmente nueva, o sobre exigir a cuerpos en situación de vulnerabilidad, la subversión. La especulación como parte de las metodologías tullidas debe de colocar señuelos epistemológicos susceptibles de presentar posibilidades en situaciones actuales. En este sentido, el trabajo de Sora y Brea son formas creativas de presentar posibilidades que permiten imaginar posibles escenarios que ya están sucediendo en el ahora y, que desbordan las expectativas de las temporalidades lineales y los espacios normativos. En suma, metodologías que permitan desarrollar contingencias que despierten el apetito por lo que es posible, por lo que podría ser o por lo que pudo haber tenido lugar.

\subsection{Descanso}

En 2015, Jennifer Brea era una estudiante de doctorado de Harvard comprometida con su pareja, cuando una misteriosa fiebre la deja postrada en cama. Progresivamente más enferma, va perdiendo la habilidad incluso de sentarse en una silla de ruedas, mientras los médicos le dicen que todo está en su cabeza. Incapaz de transmitir la gravedad y profundidad de sus sín- 
tomas, Jennifer comienza un diario en su iPhone, que eventualmente se convierte en el largometraje titulado Unrest.

Unrest (2017) es el viaje visual de una mujer diagnosticada con Síndrome de Fatiga Crónica, quien ha experimentado incertidumbre, confusión e incluso incredulidad por parte de los médicos, la comunidad y sociedad en su conjunto. En Unrest, Jennifer comparte su dolor y los momentos más íntimos de su vida apelando a lo incómodo, a la vulnerabilidad de su cuerpo enfermo. Cuerpos que como el de ella son fáciles de ignorar ya que después de todo, están demasiado enfermos para abandonar sus hogares, para salir de la cama.

El trabajo de Brea en el largometraje consiste en ir más allá de la tradicional política y denuncia de acomodar mejor a las personas con discapacidad modificado las geografías y los espacios y tiempos para hacerlos más accesibles. Brea busca un entendimiento de la normalidad de hacer simplemente las cosas de manera diferente (Hansen y Philo, 2006). En varios momentos en el documental, vemos a Brea acostada en la acera, descansando su cuerpo impregnado de dolor (Imagen 4). En otros momentos, la apreciamos retorciéndose de dolor, derrumbándose en el portal de su casa (Imagen 5), tomándose su tiempo para recuperar su movilidad, mientras su esposo la acompaña en desesperación de no poder siquiera tocarla.

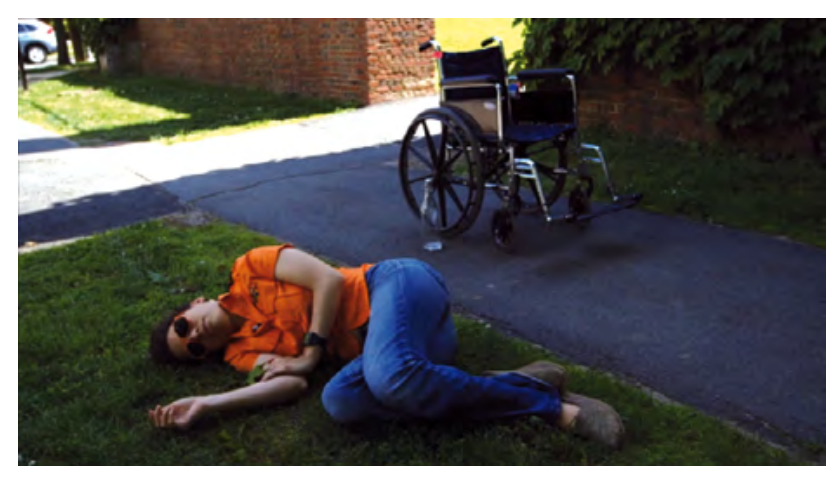

Imagen 4

Brea, Fotograma Unrest. 2017, min. 65

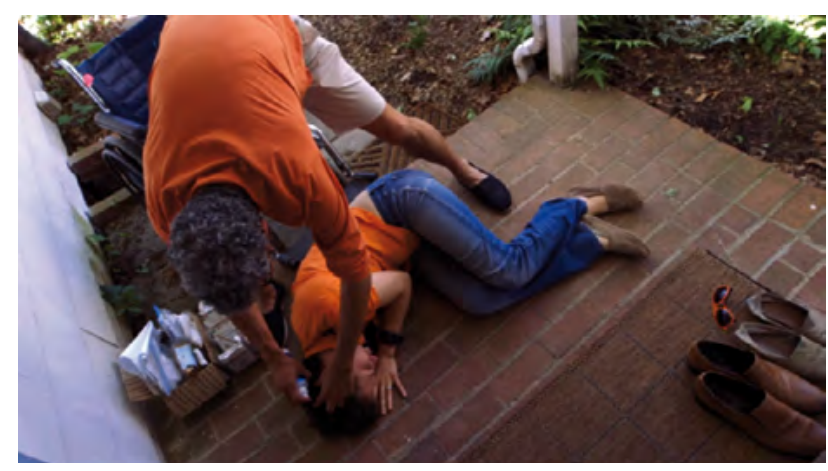

Imagen 5

Brea, Fotograma Unrest. 2017, min. 64

El uso del espacio de Brea, el posicionamiento desbordado de su cuerpo y sus gesticulaciones, es lo que Dorn (1994) enfatiza como «disidencia espacial» entre los cuerpos con dolor y la organización material de los espacios sociales, y remarca que «[r]evisionar la discapacidad como disidencia espacial es destacar su fisicalidad" (Dorn 1994: 3). La disidencia espacial es la expresión de la vulnerabilidad de los cuerpos precarios para subvertir la temporalidad y la espacialidad. En la imagen de abajo a la izquierda (Imagen 6) observamos a Brea derrumbada en la escalera de su casa. En un primer momento, la fotografía trasmite incomodidad porque su cuerpo ocupa la escalera de manera inapropiada. Un cuerpo que aparece ocupando un espacio de forma no pertinente. A su derecha, vemos la foto 35A (Imagen 7) que muestra las escaleras de la casa de Sora. Sora ha capturado las escaleras en un plano visual que proviene desde abajo como recordatorio constante de las implicaciones de transitar por este espacio que, el solo pensar en él, le genera dolor. 


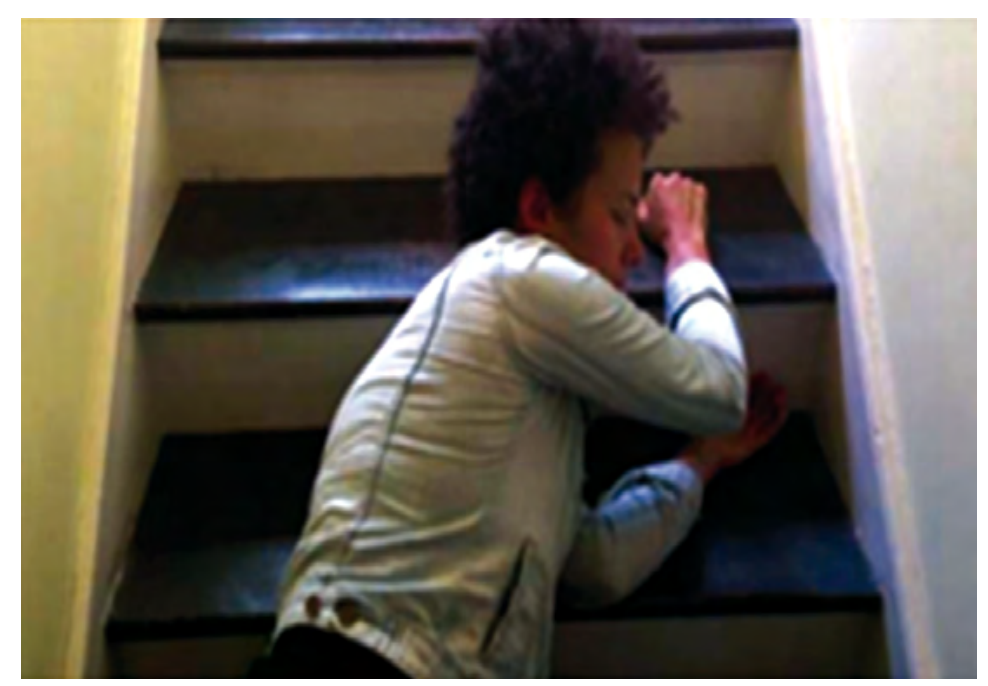

Imagen 6

Brea, Fotograma Unrest. 2017, $\min .1: 29: 28$

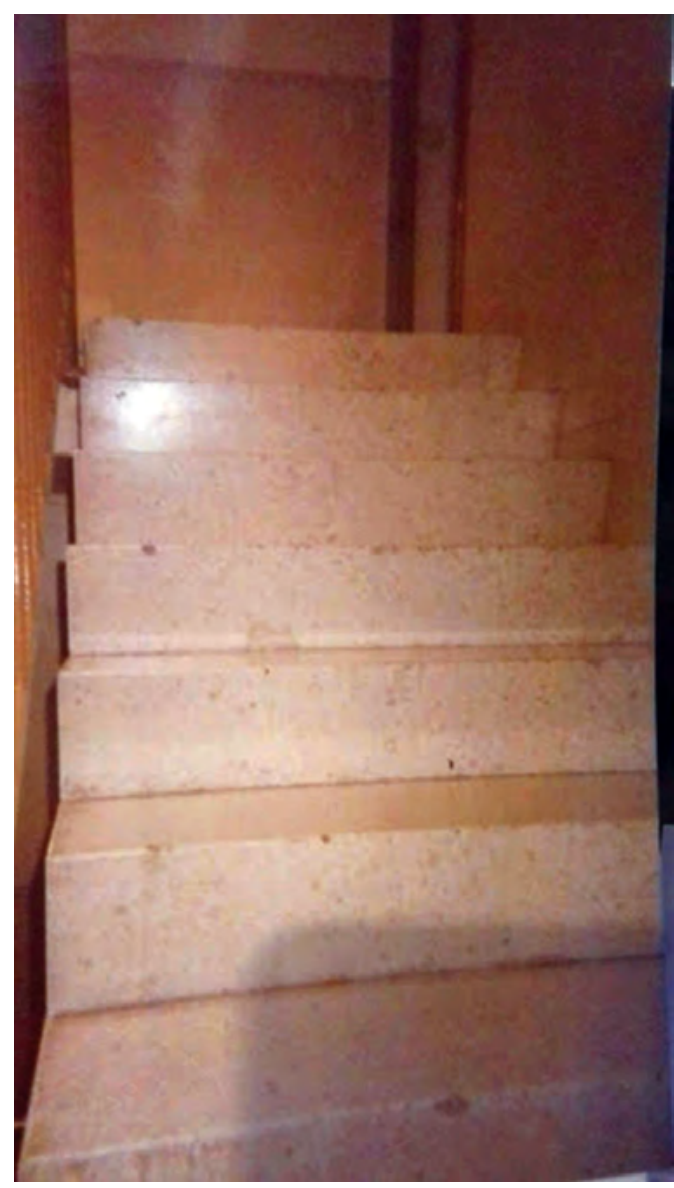

Imagen 7

Sora, Foto 35A. Escaleras 2018, $10 \mathrm{~cm} \times 15 \mathrm{~cm}$, papel

Lo interesante de la comparativa es primero observar la presencia/ausencia del cuerpo que des/aparece. En el ejercicio visual de Brea, ella pone su cuerpo para visualizar las prácticas cotidianas que le exigen negociar diariamente su dolor. Sora hace algo más revelador aún. En su fotografía "vemos» los cuerpos que faltan. Vemos aquellos cuerpos ausentes que no pueden ocupar esos espacios. Lo segundo a tomar en consideración es el propio significado de Sora sobre esta fotografía. Sora no solamente ha aprendido a mirar y a reapropiarse de los espacios, sino también de su destiempo y, con ello, legitimar nuevas fracciones temporales que ahora aparecen ante ella como tiempos que se ajustan a su cuerpo.

Simplificarse la vida conforme me levanto, y poco a poco hago lo que me deje $\mathrm{mi}$ cuerpo. Y si me toca sentarme en las escaleras de mi casa, y que me vean, no tengo porque esconder nada. Si tengo que sentarme en un banco o en las escaleras me siento. Que si incomodo, pues lo hago. Que si tienen prisa, que me brinquen. Que si mi cuerpo quiere descasar ahí, sentada, ya lo hago. He aprendido a mirar diferente a los espacios. A mi ya no me incomoda ver a alguien acostado en la calle o reposando en la escalera. La gente se molesta porque ahí no van esos cuerpos. Porque los espacios son para la gente que puede estar en ellos. Las escaleras son para la gente que puede usarlas, pero si yo no puedo ya, por qué no acostarme en ellas. Ese es el uso que yo le puedo dar a un espacio que no está pensando para mí. 
Tanto para Sora como para Brea el descanso, el parar, el frenarse, que habían sido encarnaciones de vergüenza, ahora son la oportunidad para reafirmar su fisicalidad, ocupando tiempos en disidencia. Estas temporalidades responden a lo que Carmen Sáez de Buenaventura denomina una "huelga inconsciente» (apud Távora, 2007: 3) que permite legitimar sus síntomas como una forma de decir no a los mandatos. Bajo esta perspectiva, tanto el dolor como su temporalidad, son una forma de protesta que le permite a Sora oponerse a las tensiones entre "lo que se espera de ella[s] y por otro lado no atenderlos» (ibídem: 3) y romper, por un momento, las situaciones de dependencia de las que es parte pero que, por su contexto de vulnerabilidad de género, no puede del todo dejar.

En esto consiste la política irreverente de «vivir a destiempo», en reclamar formas temporales que, aunque precarias o momentáneas, son formas legítimas. Además, recordemos que el dolor cronificado tiene una marca de género, por lo que esta serie de prácticas y experiencias corporales (asincrónicas, anacrónicas, de retrasos, de obstáculos, de demoras) también interrumpe y desborda las narraciones tradicionales de los mandatos exigentes de género. Lo anterior plantea nuevos modos de (des)ordenar las expectativas y socialización de género y una (re)valorización la experiencia del dolor exigiendo una reconceptualización de los ritmos corporales permitiendo, con ello, la posibilidad de temporalidades irreverentes que informen formas alternativas de vivir el tiempo.

\section{CONCLUSIONES}

"Vivir a destiempo" es un ejercicio crítico de pensar y cuestionar el régimen temporal normativo y violento que pone al límite al cuerpo con dolor cronificado y las posibilidades de su existencia. Este artículo propone el concepto de "vivir a destiempo" como una experiencia específica de la vida de Sora, quién vive con dolor desde los 15 años, y que, a través de un ejercicio fotográfico y narrativo, captura y materializa las temporalidades y espacios de su dolor cronificado. "Vivir a destiempo" es una temporalidad con utilidad dentro de los debates contemporáneos de las temporalidades queer y tullidas que expone una forma de vida y subjetividad que se vive en coexistencia con los marcos temporales normativos. Aunque familiarizo en gran medida con las teorias de Halberstam (2005), Esteban Muñoz (2009) y Freeman (2010), las particularidades de la vida de Sora me llevan a situar el reclamo de "vivir a destiempo" desde una mirada expandida de las epistemologías tullidas, aproximándolo a demandas, lenguajes, significaciones y contextos de corporalidades específicas.

De esta manera, "vivir a destiempo" es una temporalidad que alberga la coexistencia de tiempos, de cuerpos y de deseos con las lógicas temporales normativas siendo así una lucha política de la experiencia tullida por legitimar posibilidades de vivir con otros tiempos que buscan su expulsión y exclusión. Además, "vivir a destiempo" forma parte de un complejo sistema de opresiones de género en donde la disminución y desgaste corporal del cuerpo es resultado de una genealogía dentro de un contexto de vulnerabilidad que hace evidente y necesario nuevos planteamientos sobre la temporalidad.

Aunque sugiero un modo alternativo de conceptualizar el tiempo, esta sugerencia es más una invitación a la conversación y al debate que permita regresar, por un lado, como propone Ana Távora (2007) al cuerpo como lugar de expresión de conflictos; y, por otro lado, a la expe- 
riencia de la vida cotidiana de las mujeres con dolor cronificado como fuente epistemológica de conocimiento académico y, de esta manera, generar nuevos lenguajes tullidos y gramáticas temporales que contribuyan a los estudios de la Cultura de la discapacidad (Disability culture) o de identidad discapacitada (Disability Identity) $y$, en general, al desarrollo de las epistemologías extrañas y tullidas (Queer/Crip epistemologies) en contextos de vulnerabilidad específicos.

\section{BIBLIOGRAFÍA}

Anzaldúa, G. (1987). Borderlands. La Frontera. The New Mestiza. San Francisco: Aunt Lute Books.

Brea, J. (2015). Unrest Press Kit. Documental. Los Angeles, CA: Shella Films \& Little by Little Films distributors.

Chandler, E. (2010). Sidewalk Stories: The Troubling Task of Identification. Disability Studies Quarterly, 30(3/4). doi:10.18061/dsq.v30i3/4.1293

Cua Lim, B. (2009). Translating Time: Cinema, the Fantastic, and Temporal Critique. Durham: Duke University Press.

Dorn, M. L. (1994). Disability as spatial dissidence: a cultural geography of the stigmatized body (Tesis Doctoral inédita). The Pennsylvania State University, University Park, Pennsylvania.

Edelman, L. (2004). No Future. Queer Theory and the Death Drive. Durham \& London: Duke University Press.

Freeman, E. (2010). Time Binds. Queer Temporalities, Queer Histories. Durham \& London: Duke University Press.

Foucault, M. (2012). Vigilar y Castigar. Madrid: Biblioteca Nueva.

Grosz, E. (2004). The Nick of Time. Politics, Evolution, and the Untimely. Durham: Duke University Press, 2004.

Halberstam, J. (2005). In a Queer Time and Place. Transgender Bodies, Subcultural Lives. York \& London: New York University Press.

Hansen, N., y Philo, Ch. (2007). The Normality of Doing Things Differently: Bodies, Spaces and Disability Geography. Tijdschrift voor Economische en Sociale Geografie, 98(4), 493-506.

Haraway, D. (2013). SF: Science Fiction, Speculative Fabulation, String Figures, So Far. Ada: A Journal of Gender, New Media, and Technology, 3. doi:10.7264/N3KH0K81

Jain, L.S. (2007). Living in Prognosis: Toward an Elegiac Politics. Representations, 98(1), 77-92.

Kaja, F. (1994). Women in pain: Gender and Morbidity in Mexico. Pennsylvania: University of Pennsylvania Press.

Kafer, A. (2013). Feminist, Queer, Crip. Bloomington and Indianapolis: Indiana University Press. 
Kuppers, P. (2014). Crip Time. Tikkun, 29(4), 29-31. https://doi.org/10.1215/08879982-2810062

McRuer, R. (2006). Crip Theory: Cultural Signs of Queerness and Disability. New York: New York University Press.

Mora, E., Pujal i Llombart, M., y Albertín, P. (2017). Los contextos de vulnerabilidad de género del dolor cronificado. Revista Internacional de Sociología, 75(2), 1-12. doi: 10.3989/ ris.2017.75.2.15.60

Moraga, C. (1988). En el sueño, siempre se me recibe el río. En C. Moraga y A. Castillo (Eds.), Esta puente, mi espalda. Voces de mujeres tercermundistas en los Estados Unidos (pp. 1-16). San Francisco: Ims Press.

Muñoz, J.E. (2009). Cruising Utopia: the Then and There of Queer Futurity. Nueva York: New Your University Press.

Platero, L/R. (2014). Las políticas neoliberales contra los derechos sexuales. Fundación Betiko. Disponible en: http://fundacionbetiko.org/wp-content/uploads/2014/02/Politicasneoliberales-contra-los-derechos-sexuales-de-la-ciudadan\%C3\%ADa.pdf

Price, M. (2017). What Is a Service Animal? A Careful Rethinking. Review of Disability Studies, 13(4), 1-18.

Pujal i Llombart, M., y Mora, E. (2013). Dolor, trabajo y su diagnóstico psicosocial de género: un ejemplo. Universitas Psychologica, 12(4), 1183-1195. DOI: 0.11144/Javeriana.UPSY12-4. dtyd

Rich, A. (1996). Heterosexualidad obligatoria y existencia lesbiana (1980). Revista d'Estudis Feministes, 10, 1-42.

Sáez, J. (2017). Heterofuturibilidad. En L/R. Platero, M. Rosón y E. Ortega (Eds.), Barbarismos queer y otras esdrújulas (pp. 225-227). Barcelona: Ediciones Ballaterra.

Távora Rivero, A. (2007). El cuerpo como lugar de expresión de los conflictos. En A.M. Muñoz Muñoz, C. Gregorio Gil y A. Sánchez Espinosa (Coords.), Cuerpos de mujeres: miradas, representaciones e identidades (pp. 143-162). Granada: Universidad de Granada.

Valverde, C. (2015). De la necropolítica neoliberal a la empatía radical: Violencia discreta, cuerpos excluidos y repolitización. Barcelona: Icaria. 